Теорія Ймовір. та Матем. Статист. Вип. 81,2010
Theor. Probability and Math. Statist.

No. 81, 2010, Pages 147-158 S 0094-9000(2011)00816-2

Article electronically published on January 20, 2011

\title{
A LIMIT THEOREM FOR RANDOM FIELDS WITH A SINGULARITY IN THE SPECTRUM
}

UDC 519.21

\author{
A. YA. OLENKO AND B. M. KLYKAVKA
}

\begin{abstract}
Homogeneous isotropic random fields with singularities in spectra at nonzero frequencies are studied. This class of fields generalizes the case of random fields with long range dependence where the spectrum has a singularity at the origin. We obtain a limit theorem for integral weight functionals of the field. We also discuss the difference between this class and the long range dependence.
\end{abstract}

\section{INTRODUCTION}

Let $\xi(x), x \in \mathbb{R}^{n}$, be a real, measurable mean square continuous, wide sense homogeneous, and isotropic random field (see [1, 2]) with the zero mean and covariance function

$$
\mathbf{B}_{n}(r)=\mathbf{B}_{n}(|x|)=\mathrm{E} \xi(0) \xi(x), \quad x \in \mathbb{R}^{n},
$$

where $r=|x|=\left(\sum_{i=1}^{n} x_{i}^{2}\right)^{1 / 2}$

It is known (see, for example, [1, 2] ) that there exists a bounded nondecreasing function $\boldsymbol{\Phi}(\lambda), \lambda \geq 0$ (called the spectral function of the field $\xi(x)$ ) such that

$$
\mathbf{B}_{n}(r)=2^{\frac{n-2}{2}} \Gamma\left(\frac{n}{2}\right) \int_{0}^{\infty} \frac{J_{\frac{n-2}{2}}(r \lambda)}{(r \lambda)^{\frac{n-2}{2}}} d \boldsymbol{\Phi}(\lambda),
$$

where $J_{\nu}(z)$ is the Bessel function of the first kind and of order $\nu>-\frac{1}{2}$.

A function $\varphi(\lambda), \lambda \in \mathbb{R}^{n}$, such that

$$
\begin{gathered}
\varphi(\lambda) \in L_{1}\left(\mathbb{R}^{n}\right), \quad \varphi(\lambda) \geq 0, \\
\mathbf{B}_{n}(|x|)=\int_{\mathbb{R}^{n}} e^{i(\lambda, x)} \varphi(\lambda) d \lambda, \quad x \in \mathbb{R}^{n},
\end{gathered}
$$

is called the spectral density of the random field. The spectral density of a homogeneous and isotropic random field depends on $|\lambda|$ only. In what follows we use the same notation $\varphi(\cdot)$ for both cases $\varphi(\lambda)$ and $\varphi(|\lambda|)$. It will be clear from the context whether $\varphi(\cdot)$ is a function of several parameters or of a single argument.

For a function of a single argument, we have

$$
\begin{gathered}
\lambda^{n-1} \varphi(\lambda) \in L_{1}([0,+\infty)), \\
\boldsymbol{\Phi}(\lambda)=\frac{2 \pi^{n / 2}}{\Gamma(n / 2)} \int_{0}^{\lambda} z^{n-1} \varphi(z) d z, \quad \lambda \geq 0 .
\end{gathered}
$$

2010 Mathematics Subject Classification. Primary 60G60; Secondary 60F17.

Key words and phrases. Random fields, limit theorem, integral weight functionals, spectral functions, long range dependence.

Supported by the Swedish Institute grant SI-01424/2007. 
Various properties of random fields with long range dependence (in other words, fields with the long memory) are studied in [1, 3, 4, 5]. The spectra of such random fields have a singularity at the point $\lambda=0$. For example, the spectral density admits the following representation:

$$
\varphi(\lambda)=\frac{h_{0}(|\lambda|)}{|\lambda|^{n-\alpha}}
$$

where $h_{0}(\cdot)$ is a function defined on $\mathbb{R}^{+}:=[0,+\infty)$, continuous in a neighborhood of the origin, and such that $h_{0}(0) \neq 0$ and $h_{0}(\cdot)$ is bounded in $\mathbb{R}^{+}$.

Several limit theorems are known for integral functionals over multidimensional spheres or balls for such random fields.

Homogeneous and isotropic random fields are considered in the paper 6] for the case where the spectrum has a singularity at an arbitrary point $a$. The asymptotic behavior of the difference $\Phi(a+\lambda)-\Phi(a-\lambda)$ is studied in [6] as $\lambda \rightarrow+0$. Consider the function $\boldsymbol{\Phi}^{a}(\lambda), \lambda \geq 0$, defined by

$$
\Phi^{a}(\lambda):= \begin{cases}\Phi(a+\lambda)-\Phi(a-\lambda), & 0 \leq \lambda<a \\ \Phi(a+\lambda), & \lambda \geq a\end{cases}
$$

Then $\boldsymbol{\Phi}^{a}(\cdot)$ is a spectral function and the asymptotic behavior of $\boldsymbol{\Phi}(\lambda)$ at the point $a$ is reduced to the asymptotic behavior of $\boldsymbol{\Phi}^{a}(\lambda)$ at zero. Thus one can apply the results of [3, 4] where a duality between the asymptotic behavior of a spectral function at zero and that of the variance of integrals of the random field over a sphere or a ball whose radius increases to infinity is proved.

Introducing the notation

$$
\tilde{b}^{a}(r):=(2 \pi)^{n} \int_{0}^{\infty} \frac{J_{n / 2}^{2}(r \lambda)}{(r \lambda)^{n}} d \Phi^{a}(\lambda),
$$

it is proved in [6] that there exists a real radial function $f_{n, r, a}(\cdot)$ such that

$$
\tilde{b}^{a}(r)=\operatorname{Var}\left[\int_{\mathbb{R}^{n}} f_{n, r, a}(|x|) \xi(x) d x\right]=(2 \pi)^{n} \int_{0}^{\infty} \frac{J_{n / 2}^{2}(r(\lambda-a))}{(r(\lambda-a))^{n}} d \Phi(\lambda) .
$$

A particular case of a field with long range dependence is given by the case where $a=0, \boldsymbol{\Phi}^{a}(\lambda)=\boldsymbol{\Phi}(\lambda)$, and

$$
f_{n, r, 0}(|x|)= \begin{cases}r^{-n}, & |x|<r \\ 0, & |x| \geq r\end{cases}
$$

see [6]. In this case,

$$
\int_{\mathbb{R}^{n}} f_{n, r, 0}(|x|) \xi(x) d x=\frac{1}{r^{n}} \int_{v_{n}(r)} \xi(x) d x,
$$

where $v_{n}(r)=\left\{x \in \mathbb{R}^{n}:|x| \leq r\right\}$ is the ball of radius $r$ in $\mathbb{R}^{n}$.

It was also proved in [6] that the weight function $f_{n, r, a}(|x|)$ admits the following representation:

$$
f_{n, r, a}(|x|)=\frac{1}{|x|^{n / 2-1}} \int_{0}^{\infty} \lambda^{n / 2} \frac{J_{n / 2}(r(\lambda-a))}{(r(\lambda-a))^{n / 2}} J_{n / 2-1}(|x| \lambda) d \lambda, \quad|x| \neq r .
$$

We are interested in studying the asymptotic behavior as $r \rightarrow+\infty$ of the following integral functional:

$$
\int_{\mathbb{R}^{n}} f_{n, r t^{1 / n}, a}(|x|) \xi(x) d x .
$$


We consider the random fields whose spectra $\boldsymbol{\Phi}(\lambda)$ have singularities at a point $\lambda=a \neq 0$, that is, on the sphere

$$
S_{n}(a)=\left\{x \in \mathbb{R}^{n}:|x|=a\right\} .
$$

In contrast to the case of random fields with long range dependence, the correlation functions of the fields satisfying the above property oscillate with decreasing amplitudes.

This is demonstrated by the following example.

Example. Let $n=3$ and $\alpha=\frac{1}{2}$. Consider a random field with long range dependence whose spectral density is given by

$$
\varphi(\lambda)= \begin{cases}1 / \lambda^{3-\alpha}, & 0 \leq \lambda \leq 1 \\ 0, & \lambda>1\end{cases}
$$

According to (1), (2), and

we have

$$
J_{1 / 2}(z)=\sqrt{\frac{2}{\pi}} \frac{\sin (z)}{\sqrt{z}}
$$

$$
B_{3}(r)=\frac{(2 \pi)^{3 / 2}}{\sqrt{r}} \int_{0}^{1} \frac{J_{1 / 2}(r \lambda)}{\lambda} d \lambda=\frac{4 \pi}{r} \int_{0}^{1} \frac{\sin (r \lambda)}{\lambda^{3 / 2}} d \lambda
$$

(see [7]). The function $B_{3}(r)$ depicted in Figure 1a is decreasing and does not oscillate.

Suppose another random field has a singularity at the point $\lambda=\frac{1}{2}$ and its spectral density is given by

$$
\varphi(\lambda)= \begin{cases}\frac{1}{\sqrt{\left|\lambda-\frac{1}{2}\right|}}, & 0 \leq \lambda \leq 1 \\ 0, & \lambda>1\end{cases}
$$

Then the correlation function of this random field is such that

$$
\widetilde{B}_{3}(r)=\frac{(2 \pi)^{3 / 2}}{\sqrt{r}} \int_{0}^{1} \frac{J_{1 / 2}(r \lambda) \lambda^{3 / 2}}{\sqrt{\left|\lambda-\frac{1}{2}\right|}} d \lambda=\frac{4 \pi}{r} \int_{0}^{1} \frac{\lambda \sin (r \lambda)}{\sqrt{\left|\lambda-\frac{1}{2}\right|}} d \lambda .
$$

The function $\widetilde{B}_{3}(r)$ depicted in Figure 1 b has an oscillating behavior and its amplitude is decreasing.

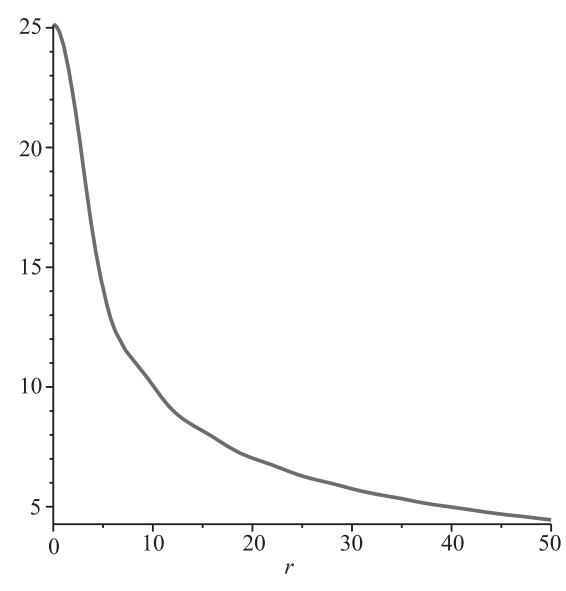

Figure 1a

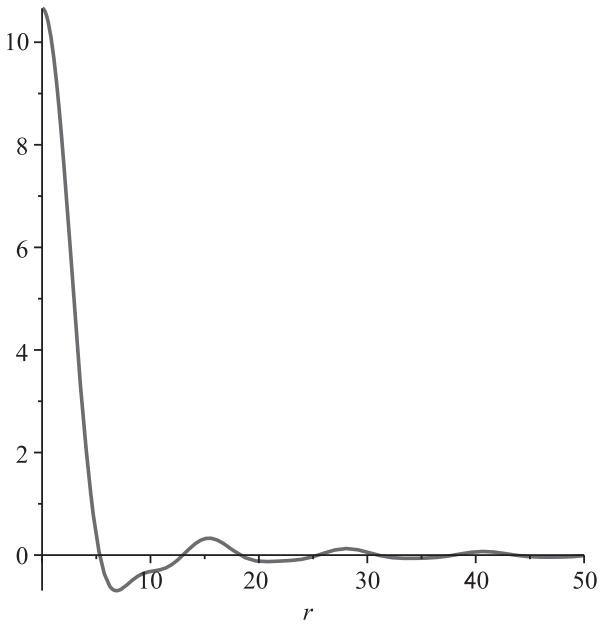

Figure 1b 


\section{MAin RESUlt}

We need an analog of representation (3) for the spectral density corresponding to the spectrum $\boldsymbol{\Phi}(\lambda)$ with a singularity at a point $a$.

Condition A. $\xi: \Omega \times \mathbb{R}^{n} \rightarrow \mathbb{R}$ is a mean square continuous homogeneous isotropic Gaussian random field whose spectral density is

$$
\varphi(\lambda)=\frac{h(|\lambda|-a)}{|| \lambda|-a|^{1-\alpha}}
$$

where $\varphi(\lambda) \in L_{1}\left(\mathbb{R}^{n}\right), \alpha>0$, and $h(\cdot)$ is a function defined in the interval $[-a,+\infty)$, continuous in a neighborhood of 0 and such that $h(0) \neq 0$ and $h(\cdot)$ is bounded in $[-a,+\infty)$.

Similarly to the case of the function $\varphi(\cdot)$, we use the same notation $h(\cdot)$ for both cases $h(\lambda)$ and $h(|\lambda|)$.

Remark 1. In what follows, we consider the case where the function $h(\cdot)$ is continuous in a neighborhood of 0 and where the index $\alpha$ in representation (4) is the same for both cases $|\lambda|>a$ and $|\lambda|<a$. Nevertheless all the results can be generalized to the case where the function $h(\cdot)$ has distinct left and right limits at zero as well as to the case where the number $\alpha$ is different for $|\lambda|>a$ and $|\lambda|<a$.

Remark 2. Comparing with the case of stochastic processes (case of $n=1$ ), the singularities of spectra for random fields are essentially different. The spectra of the stochastic processes may only have a singularity at a unique point. In the case of random fields, the singularity is present at a unique point only if $a=0$. Otherwise (that is, if $a \neq 0$ ) the spectral density has singularities at all points of the $n$-dimensional ball $S_{n}(a)$.

Remark 3. In contrast to the case of random fields with long range dependence, the index in the representation (4) of the spectral density is equal to $1-\alpha$, as compared to the index $n-\alpha$ in (3). This is explained by that the spectral density in representation (3) is integrable if the following integral (having a singularity at zero)

$$
\int_{0}^{+\infty} \lambda^{n-1} \varphi(\lambda) d \lambda=\int_{0}^{+\infty} \frac{h_{0}(\lambda)}{\lambda^{1-\alpha}} d \lambda
$$

is finite. The latter integral appears after the spherical change of variables.

The spectral density in (4) is integrable for $a>0$ if the following integral (having a singularity at the point $a$ )

$$
\int_{0}^{+\infty} \lambda^{n-1} \varphi(\lambda) d \lambda=\int_{0}^{+\infty} \lambda^{n-1} \frac{h(|\lambda|-a)}{|| \lambda|-a|^{1-\alpha}} d \lambda
$$

is finite.

In both cases, the integral of the spectral density is finite only if $\alpha>0$. The difference between the indices is explained by that the spectral density for the case of long range dependence (i.e., for the case of $a=0$ ) has a singularity at a unique point $\lambda=0$, while the spectral density has singularities at all points of the $n$-dimensional ball $S_{n}(a)$ if $a>0$.

Theorem 1. Assume that condition A holds and that $0<\alpha<1$. Then the finitedimensional distributions of the process

$$
X_{r}(t)=\frac{\sqrt{t} r^{\alpha / 2}}{(2 \pi)^{n / 2} \sqrt{2 h(0)} a^{(n-1) / 2}} \int_{\mathbb{R}^{n}} f_{n, r t^{1 / n}, a}(|x|) \xi(x) d x
$$


weakly converge as $r \rightarrow \infty$ to the finite-dimensional distributions of the process

$$
X(t)=\int_{\mathbb{R}^{n}} \frac{J_{n / 2}\left(|u| t^{1 / n}\right)}{|u|^{n-\alpha / 2}} d Z(u),
$$

where $Z(\cdot)$ is the white noise in $\left(\mathbb{R}^{n}, \mathfrak{B}^{n}\right)$.

\section{Proof of the theorem}

We use the spectral decomposition of the random field

$$
\xi(x)=\int_{\mathbb{R}^{n}} e^{i\langle\lambda, x\rangle} \sqrt{\varphi(|\lambda|)} d W(\lambda)
$$

(see [1]), where $W(\cdot)$ is the Wiener measure in $\left(\mathbb{R}^{n}, \mathfrak{B}^{n}\right)$.

Consider the following integral of the random field:

$$
\int_{\mathbb{R}^{n}} f_{n, r, a}(|x|) \xi(x) d x
$$

Substituting the spectral decomposition (5) for $\xi(x)$ in the integral (6), we get

$$
\begin{aligned}
& \int_{\mathbb{R}^{n}} f_{n, r, a}(x) \int_{\mathbb{R}^{n}} e^{i\langle\lambda, x\rangle} \sqrt{\varphi(|\lambda|)} d W(\lambda) d x \\
& \quad \stackrel{d}{=} \int_{\mathbb{R}^{n}} \int_{\mathbb{R}^{n}} f_{n, r, a}(x) e^{i\langle\lambda, x\rangle} d x \sqrt{\varphi(|\lambda|)} d W(\lambda) .
\end{aligned}
$$

To justify the transformation in (7), we note that

$$
\int_{\mathbb{R}^{n}} e^{i\langle\lambda, x\rangle} \sqrt{\varphi(|\lambda|)} d W(\lambda)
$$

is the Fourier transform of the stochastic measure $\mu(d \lambda)=\sqrt{\varphi(|\lambda|)} d W(\lambda)$, while

$$
\int_{\mathbb{R}^{n}} f_{n, r, a}(x) e^{i\langle\lambda, x\rangle} d x
$$

is the usual Fourier transform of the function $f_{n, r, a}(x)$. Thus equality (7) takes the form

$$
\int_{\mathbb{R}^{n}} f_{n, r, a}(x) d \widehat{\mu}(x) \stackrel{d}{=} \int_{\mathbb{R}^{n}} \widehat{f}_{n, r, a}(\lambda) d \mu(\lambda),
$$

which is a stochastic analogue of the Parseval equality. Since $f_{n, r, a}(\cdot) \in L_{2}\left(\mathbb{R}^{n}\right)$ (see [6]) and $\sqrt{\varphi(|\cdot|)} \in L_{2}\left(\mathbb{R}^{n}\right)$, equality (17) follows; see [8].

The function $f_{n, r, a}(|x|)$ is defined in [6] as a solution of the equation

$$
\int_{\mathbb{R}^{n}} f_{n, r, a}(|x|) e^{i\langle\lambda, x\rangle} d x=(2 \pi)^{n / 2} \frac{J_{n / 2}(r(|\lambda|-a))}{(r(|\lambda|-a))^{n / 2}} .
$$

Substituting (8) in (7), we obtain

$$
\begin{aligned}
\int_{\mathbb{R}^{n}} & \int_{\mathbb{R}^{n}} f_{n, r, a}(x) e^{i\langle\lambda, x\rangle} d x \sqrt{\varphi(|\lambda|)} d W(\lambda) \\
& \stackrel{d}{=}(2 \pi)^{n / 2} \int_{\mathbb{R}^{n}} \frac{J_{n / 2}(r(|\lambda|-a))}{(r(|\lambda|-a))^{n / 2}} \sqrt{\varphi(|\lambda|)} d W(\lambda) .
\end{aligned}
$$

The ratio $J_{\nu}(x) / x^{\nu}$ is an even function. If the spectrum is absolutely continuous, the finite-dimensional distributions of the stochastic integral do not change under the change of the integrand on a set of zero Lebesgue measure. 
Thus the integral in (9) is the sum of two integrals,

$$
\begin{aligned}
(2 \pi)^{n / 2} & \int_{\mathbb{R}^{n}} \frac{J_{n / 2}(r(|\lambda|-a))}{(r(|\lambda|-a))^{n / 2}} \sqrt{\varphi(|\lambda|)} W(d \lambda) \\
\stackrel{d}{=} & (2 \pi)^{n / 2} \int_{|\lambda|>a} \frac{J_{n / 2}(r(|\lambda|-a))}{(r(|\lambda|-a))^{n / 2}} \sqrt{\varphi(|\lambda|)} d W(\lambda) \\
& +(2 \pi)^{n / 2} \int_{|\lambda|<a} \frac{J_{n / 2}(r(a-|\lambda|))}{(r(a-|\lambda|))^{n / 2}} \sqrt{\varphi(|\lambda|)} d W(\lambda) \\
= & : I_{1}(r)+I_{2}(r) .
\end{aligned}
$$

In the integral $I_{1}(r)$, we change the variables

$$
\begin{cases}u=\lambda\left(1-\frac{a}{|\lambda|}\right), & |u|=|\lambda|-a, \\ \lambda=u\left(1+\frac{a}{|u|}\right), & |\lambda|=|u|+a .\end{cases}
$$

Note that this transformation is bijective and maps the set

$$
\mathbb{R}^{n} \backslash v_{n}(a)=\left\{\lambda \in \mathbb{R}^{n}:|\lambda|>a\right\}
$$

into the set

$$
\mathbb{R}_{0}^{n}:=\mathbb{R}^{n} \backslash\{(0, \ldots, 0)\}
$$

Since

$$
\lambda_{i}=u_{i}+a \frac{u_{i}}{|u|}=u_{i}+a \frac{u_{i}}{\sqrt{\sum_{i=1}^{n} u_{i}^{2}}}
$$

in (10), we have

$$
\begin{gathered}
\frac{\partial \lambda_{i}}{\partial u_{i}}=1+a \frac{|u|^{2}-u_{i}^{2}}{|u|^{3}}, \\
\frac{\partial \lambda_{i}}{\partial u_{j}}=-a \frac{u_{i} u_{j}}{|u|^{3}}, \quad i \neq j .
\end{gathered}
$$

Hence the Jacobian matrix of transformation (10) is given by

$$
\begin{aligned}
J_{n}(u) & =\left(\frac{\partial \lambda_{i}}{\partial u_{j}}\right)_{i, j=1}^{n} \\
& =\frac{1}{|u|^{3}}\left(\begin{array}{cccc}
|u|^{3}+a\left(|u|^{2}-u_{1}^{2}\right) & -a u_{1} u_{2} & \ldots & -a u_{1} u_{n} \\
-a u_{2} u_{1} & |u|^{3}+a\left(|u|^{2}-u_{2}^{2}\right) & \ldots & \ldots \\
\ldots & \ldots & \ldots & \ldots \\
-a u_{n} u_{1} & \ldots & \ldots & |u|^{3}+a\left(|u|^{2}-u_{n}^{2}\right)
\end{array}\right) .
\end{aligned}
$$

Lemma 1. The Jacobian matrix of transformation (10) is equal to

$$
\operatorname{det}\left(J_{n}(u)\right)=\left(1+\frac{a}{|u|}\right)^{n-1}, \quad|u| \neq 0 .
$$

Proof. Note that transformation (10) is radial. Thus for all pairs of vectors $\lambda^{1}$ and $\lambda^{2}$ whose lengths are the same, i.e., $\left|\lambda^{1}\right|=\left|\lambda^{2}\right|$, the scaled images are the same in a neighborhood of these vectors. This means that the Jacobian determinant is the same for all vectors of the same length. This allows us to evaluate it along a single direction chosen appropriately. The calculations simplify if we consider the direction $\left(u_{1}, 0, \ldots, 0\right)$. We have

$$
J_{n}(u)=\frac{1}{|u|^{3}}\left(\begin{array}{cccc}
|u|^{3} & 0 & \ldots & 0 \\
0 & |u|^{3}+a|u|^{2} & \ldots & 0 \\
0 & \cdots & \ldots & 0 \\
0 & 0 & 0 & |u|^{3}+a|u|^{2}
\end{array}\right) .
$$


Thus

$$
\operatorname{det}\left(J_{n}(u)\right)=\frac{1}{|u|^{3 n}}|u|^{3}\left(|u|^{3}+a|u|^{2}\right)^{n-1}=\left(1+\frac{a}{|u|}\right)^{n-1} .
$$

We apply the formula for the change of variables in the stochastic integral, 9, Proposition 4.2], 10. Theorem 4.4], and Lemma 1. Note that [9, Proposition 4.2] and [10. Theorem 4.4] are simpler for our case, since we deal with a single function:

$$
\begin{aligned}
I_{1}(r) & =(2 \pi)^{n / 2} \int_{|\lambda|>a} \frac{J_{n / 2}(r(|\lambda|-a))}{(r(|\lambda|-a))^{n / 2}} \sqrt{\frac{h(|\lambda|-a)}{(|\lambda|-a)^{1-\alpha}}} d W(\lambda) \\
& \stackrel{d}{=}(2 \pi)^{n / 2} \int_{\mathbb{R}_{0}^{n}} \frac{J_{n / 2}(r|u|)}{(r|u|)^{n / 2}} \sqrt{\frac{h(|u|)}{|u|^{1-\alpha}}} d W\left(u\left(1+\frac{a}{|u|}\right)\right) \\
& \stackrel{d}{=}(2 \pi)^{n / 2} \int_{\mathbb{R}_{0}^{n}} \frac{J_{n / 2}(r|u|)}{(r|u|)^{n / 2}} \sqrt{\frac{h(|u|)}{|u|^{1-\alpha}}} \sqrt{\operatorname{det}\left(J_{n}(u)\right)} d \widetilde{W}(u) \\
& \stackrel{d}{=}(2 \pi)^{n / 2} \int_{\mathbb{R}^{n}} \frac{J_{n / 2}(r|u|)}{(r|u|)^{n / 2}} \sqrt{\frac{h(|u|)}{|u|^{1-\alpha}}\left(1+\frac{a}{|u|}\right)^{n-1}} d \widetilde{W}(u),
\end{aligned}
$$

where $\widetilde{W}(\cdot)$ is the Wiener measure in $\left(\mathbb{R}^{n}, \mathfrak{B}^{n}\right)$.

Now we change the variables $r u_{i}=\widetilde{u}_{i}, i=1, \ldots, n$, in the latter integral and use the property that a Gaussian white noise is semistable of order $n / 2$ :

$$
d W(c x)=c^{n / 2} d W(x) .
$$

Then the integral $I_{1}(\cdot)$ depends on the argument $r t^{1 / n}, t \in[0,1]$, namely

$$
\begin{aligned}
& I_{1}\left(r t^{1 / n}\right) \\
& \quad \stackrel{d}{=}(2 \pi)^{n / 2} \frac{r^{(1-\alpha-n) / 2}}{\sqrt{t}} \int_{\mathbb{R}^{n}} \frac{J_{n / 2}\left(|\widetilde{u}| t^{1 / n}\right)}{(|\widetilde{u}|)^{n / 2}} \sqrt{\frac{h(|\widetilde{u}| / r)}{|\widetilde{u}|^{1-\alpha}}\left(1+\frac{a r}{|\widetilde{u}|}\right)^{n-1}} d \widetilde{W}(\widetilde{u}) .
\end{aligned}
$$

Further asymptotic behavior of the integral $I_{1}\left(r t^{1 / n}\right)$ is studied similarly to the proof of Theorem 2.10.1 in [1].

Let

$$
Y(t):=\int_{\mathbb{R}^{n}} \frac{J_{n / 2}\left(|\widetilde{u}| t^{1 / n}\right)}{|\widetilde{u}|^{n-\alpha / 2}} d \widetilde{W}(\widetilde{u}), \quad t \in[0,1] .
$$

Relations (12) and (13) imply that

$$
\begin{aligned}
R_{r}(t) & :=\mathrm{E}\left(\frac{\sqrt{t} r^{\alpha / 2}}{(2 \pi)^{n / 2} \sqrt{h(0)} a^{(n-1) / 2}} I_{1}\left(r t^{1 / n}\right)-Y(t)\right)^{2} \\
& =\int_{\mathbb{R}^{n}} \frac{J_{n / 2}^{2}\left(|\widetilde{u}| t^{1 / n}\right)}{|\widetilde{u}|^{2 n-\alpha}} Q_{r}(|\widetilde{u}|) d \widetilde{u}
\end{aligned}
$$

where

$$
Q_{r}(|\widetilde{u}|):=\left(\sqrt{\frac{h(|\widetilde{u}| / r)}{h(0)}\left(1+\frac{|\widetilde{u}|}{a r}\right)^{n-1}}-1\right)^{2} .
$$

Let $\psi(r) \rightarrow \infty$ and $\psi(r) / r \rightarrow 0$ as $r \rightarrow \infty$. We represent the integral in (14) as the sum of two integrals, $R_{r}(t)=R_{r, 1}(t)+R_{r, 2}(t)$. The domains of integration are $B_{1}:=\left\{\widetilde{u} \in \mathbb{R}^{n}:|\widetilde{u}| \leq \psi(r)\right\}$ for $R_{r, 1}(t)$ and $B_{2}:=\left\{\widetilde{u} \in \mathbb{R}^{n}:|\widetilde{u}|>\psi(r)\right\}$ for $R_{r, 2}(t)$. 
According to condition A, for every $\varepsilon>0$ there exists $r_{0}$ such that $Q_{r}(|\widetilde{u}|)<\varepsilon$ for $r>r_{0}$ and $\widetilde{u} \in B_{1}$. Since

$$
\begin{aligned}
& \frac{J_{n / 2}^{2}(s)}{s^{n}} \sim \frac{1}{2^{n} \Gamma^{2}\left(\frac{n}{2}+1\right)} \quad \text { as } s \rightarrow 0, \\
& \frac{J_{n / 2}^{2}(s)}{s^{n}}=O\left(\frac{1}{s^{n+1}}\right) \quad \text { as } s \rightarrow+\infty
\end{aligned}
$$

(see [7]), the integral

$$
\begin{aligned}
\int_{\mathbb{R}^{n}} \frac{J_{n / 2}^{2}\left(|\widetilde{u}| t^{1 / n}\right)}{|\widetilde{u}|^{2 n-\alpha}} d \widetilde{u} & =\left|u=t^{1 / n} \widetilde{u}\right|=\int_{\mathbb{R}^{n}} \frac{J_{n / 2}^{2}(|u|)}{|u|^{2 n-\alpha}} t^{1-\alpha / n} d u \\
& =\frac{2 \pi^{n / 2} t^{1-\alpha / n}}{\Gamma(n / 2)} \int_{0}^{\infty} \frac{J_{n / 2}^{2}(s)}{s^{n+1-\alpha}} d s
\end{aligned}
$$

is uniformly bounded in $t$.

Thus we can make $R_{r, 1}(t)$ as small as we want by decreasing $\varepsilon$.

The Bessel functions are bounded, that is, $\left|J_{n / 2}(u)\right| \leq 1$ (see [7), thus there exists $r^{0}$ such that the second integral can be estimated as follows:

$$
\begin{aligned}
R_{r, 2}(t) & \leq \frac{\sup _{u \in[-a,+\infty)} h(u)}{h(0)} \int_{B_{2}} \frac{J_{n / 2}^{2}\left(|\widetilde{u}| t^{1 / n}\right)}{|\widetilde{u}|^{2 n-\alpha}}(1+|\widetilde{u}|)^{n-1} d \widetilde{u} \\
& =O\left(\int_{B_{2}} \frac{d \widetilde{u}}{|\widetilde{u}|^{1-\alpha+n}}\right)=O\left(\int_{\psi(r)}^{+\infty} \frac{d s}{s^{2-\alpha}}\right)
\end{aligned}
$$

for $r>r^{0}$. The latter expression approaches zero as $r \rightarrow+\infty$. Hence $\lim _{r \rightarrow \infty} R_{r}(t)=0$ and

$$
\lim _{r \rightarrow+\infty} \mathrm{E} \sum_{j=1}^{p} a_{j}\left(\frac{\sqrt{t_{j}} r^{\alpha / 2}}{(2 \pi)^{n / 2} \sqrt{h(0)} a^{(n-1) / 2}} I_{1}\left(r t_{j}^{1 / n}\right)-Y\left(t_{j}\right)\right)^{2}=0
$$

for all $a_{j} \in \mathbb{R}$ and $t_{j} \in[0,1], j=1, \ldots, p$. This implies the convergence as $r \rightarrow+\infty$ of the finite-dimensional distributions of the integral

$$
\frac{\sqrt{t} r^{\alpha / 2}}{(2 \pi)^{n / 2} \sqrt{h(0)} a^{(n-1) / 2}} I_{1}\left(r t^{1 / n}\right)
$$

to those of the process $Y(t), t \in[0,1]$.

The integral $I_{2}(r)$ is considered similarly. Since the function $J_{n / 2}(x) / x^{n / 2}$ is even, the integral admits the following representation:

$$
I_{2}(r)=(2 \pi)^{n / 2} \int_{|\lambda|<a} \frac{J_{n / 2}(r(a-|\lambda|))}{(r(a-|\lambda|))^{n / 2}} \sqrt{\varphi(|\lambda|)} d W(\lambda) .
$$

Now we change the variables

$$
\begin{cases}u=\lambda\left(\frac{a}{|\lambda|}-1\right), & |u|=a-|\lambda|, \\ \lambda=u\left(\frac{a}{|u|}-1\right), & |\lambda|=a-|u| .\end{cases}
$$

This transformation is a bijection of the ball with center removed, $\left\{\lambda \in \mathbb{R}^{n}: 0<|\lambda|<a\right\}$, to itself. 
Relation (15) implies that

$$
\begin{gathered}
\lambda_{i}=a \frac{u_{i}}{\sqrt{\sum_{i=1}^{n} u_{i}^{2}}}-u_{i}, \\
\frac{\partial \lambda_{i}}{\partial u_{i}}=a \frac{|u|^{2}-u_{i}^{2}}{|u|^{3}}-1, \\
\frac{\partial \lambda_{i}}{\partial u_{j}}=-a \frac{u_{i} u_{j}}{|u|^{3}}, \quad i \neq j .
\end{gathered}
$$

Thus the Jacobian matrix of transformation (15) is equal to

$$
\begin{aligned}
\widetilde{J}_{n}(u) & =\left(\frac{\partial \lambda_{i}}{\partial u_{j}}\right)_{i, j=1}^{n} \\
& =\frac{1}{|u|^{3}}\left(\begin{array}{cccc}
a\left(|u|^{2}-u_{1}^{2}\right)-|u|^{3} & -a u_{1} u_{2} & \ldots & -a u_{1} u_{n} \\
-a u_{2} u_{1} & a\left(|u|^{2}-u_{2}^{2}\right)-|u|^{3} & \ldots & \ldots \\
\ldots & \ldots & \ldots & \ldots \\
-a u_{n} u_{1} & \ldots & \ldots & a\left(|u|^{2}-u_{n}^{2}\right)-|u|^{3}
\end{array}\right) .
\end{aligned}
$$

Lemma 2. The Jacobian determinant of transformation (15) is equal to

$$
\operatorname{det}\left(\widetilde{J}_{n}(u)\right)=-\left(\frac{a}{|u|}-1\right)^{n-1}, \quad|u| \neq 0 .
$$

Proof. Similarly to the proof of Lemma 1, it is sufficient to consider the Jacobian matrix only for the direction $\left(u_{1}, 0, \ldots, 0\right)$ :

$$
\widetilde{J}_{n}(u)=\frac{1}{|u|^{3}}\left(\begin{array}{cccc}
-|u|^{3} & 0 & \cdots & 0 \\
0 & a|u|^{2}-|u|^{3} & \cdots & 0 \\
0 & \cdots & \cdots & 0 \\
0 & 0 & 0 & a|u|^{2}-|u|^{3}
\end{array}\right) .
$$

Thus

$$
\operatorname{det}\left(\widetilde{J}_{n}(u)\right)=-\frac{|u|^{3}}{|u|^{3 n}}\left(a|u|^{2}-|u|^{3}\right)^{n-1}=-\left(\frac{a}{|u|}-1\right)^{n-1}
$$

Applying the formula for the change of variables in the stochastic integral together with Lemma 2, we get

$$
I_{2}(r) \stackrel{d}{=}(2 \pi)^{n / 2} \int_{|u|<a} \frac{J_{n / 2}(r|u|)}{(r|u|)^{n / 2}} \sqrt{\frac{h(-|u|)}{|u|^{1-\alpha}}\left(\frac{a}{|u|}-1\right)^{n-1}} d \bar{W}(u),
$$

where $\bar{W}(\cdot)$ is Wiener measure in $\left(\mathbb{R}^{n}, \mathfrak{B}^{n}\right)$, which is independent of $\widetilde{W}(\cdot)$.

Making the change of variables $r u_{i}=\widetilde{u}_{i}, i=1, \ldots, n$, in the latter integral and using the semistability of the Gaussian white noise (11), we prove that

$$
\begin{aligned}
& I_{2}\left(r t^{1 / n}\right) \\
& \quad \stackrel{d}{=}(2 \pi)^{n / 2} \frac{r^{\frac{1-\alpha-n}{2}}}{\sqrt{t}} \int_{|\widetilde{u}|<r a} \frac{J_{n / 2}\left(|\widetilde{u}| t^{1 / n}\right)}{(|\widetilde{u}|)^{n / 2}} \sqrt{\frac{h\left(-\frac{|\widetilde{u}|}{r}\right)}{|\widetilde{u}|^{1-\alpha}}\left(\frac{a r}{|\widetilde{u}|}-1\right)^{n-1} d \bar{W}(\widetilde{u}) .}
\end{aligned}
$$

Now let

$$
V(t):=\int_{\mathbb{R}^{n}} \frac{J_{n / 2}\left(|\widetilde{u}| t^{1 / n}\right)}{|\widetilde{u}|^{n-\alpha / 2}} d \bar{W}(\widetilde{u}), \quad t \in[0,1]
$$


Relations (16) and (17) imply that

$$
\begin{aligned}
R_{r}^{1}(t) & :=\mathrm{E}\left(\frac{\sqrt{t} r^{\alpha / 2}}{(2 \pi)^{n / 2} \sqrt{h(0)} a^{(n-1) / 2}} I_{2}\left(r t^{1 / n}\right)-V(t)\right)^{2} \\
& =\int_{\mathbb{R}^{n}} \frac{J_{n / 2}^{2}\left(|\widetilde{u}| t^{1 / n}\right)}{|\widetilde{u}|^{2 n-\alpha}} Q_{r}^{1}(|\widetilde{u}|) d \widetilde{u}
\end{aligned}
$$

where

$$
\begin{gathered}
Q_{r}^{1}(|\widetilde{u}|):=\left(\sqrt{\frac{h(-|\widetilde{u}| / r)}{h(0)}\left(1-\frac{|\widetilde{u}|}{a r}\right)_{+}^{n-1}}-1\right)^{2}, \\
(x)_{+}= \begin{cases}x & \text { if } x \geq 0, \\
0 & \text { if } x<0 .\end{cases}
\end{gathered}
$$

As in the case of $R_{r}(t)$, we represent the integral on the right hand side of (18) as the sum of two integrals, $R_{r}^{1}(t)=R_{r, 1}^{1}(t)+R_{r, 2}^{1}(t)$, whose domains of integration are $B_{1}$ for $R_{r, 1}^{1}(t)$ and $B_{2}$ for $R_{r, 2}^{1}(t)$.

Similarly to what we did for $R_{r, 1}(t)$ we prove that $R_{r, 1}^{1}(t)$ can be made as small as we want uniformly in $t \in[0,1]$.

For the integral $R_{r, 2}^{1}(t)$, we use the estimate $\left|J_{n / 2}(u)\right| \leq 1$ and note that

$$
Q_{r}^{1}(|\widetilde{u}|) \leq \frac{\sup _{u \in[-a,+\infty)} h(u)}{h(0)} .
$$

This yields

$$
\begin{aligned}
R_{r, 2}^{1}(t) & \leq \frac{\sup _{u \in[-a,+\infty)} h(u)}{h(0)} \int_{B_{2}} \frac{J_{n / 2}^{2}\left(|\widetilde{u}| t^{1 / n}\right)}{|\widetilde{u}|^{2 n-\alpha}} d \widetilde{u} \\
& =O\left(\int_{B_{2}} \frac{d \widetilde{u}}{|\widetilde{u}|^{2 n-\alpha}}\right)=O\left(\int_{\psi(r)}^{+\infty} \frac{d s}{s^{n+1-\alpha}}\right) .
\end{aligned}
$$

The latter expression approaches zero as $r \rightarrow+\infty$.

Similarly to the proof for $I_{1}$, the above reasoning implies the convergence as $r \rightarrow+\infty$ of finite-dimensional distributions of the integral

$$
\frac{\sqrt{t} r^{\alpha / 2}}{(2 \pi)^{n / 2} \sqrt{h(0)} a^{(n-1) / 2}} I_{2}\left(r t^{1 / n}\right)
$$

to those of the process $V(t), t \in[0,1]$. Thus the finite-dimensional distributions of the process

$$
\sqrt{2} X_{r}(t) \stackrel{d}{=} \frac{\sqrt{t} r^{\alpha / 2}}{(2 \pi)^{n / 2} \sqrt{h(0)} a^{(n-1) / 2}}\left(I_{1}\left(r t^{1 / n}\right)+I_{2}\left(r t^{1 / n}\right)\right)
$$

converge to those of the process

$$
Y(t)+V(t), \quad t \in[0,1] .
$$

Since the Wiener measures $\widetilde{W}(\cdot)$ and $\bar{W}(\cdot)$ are independent, there exists another Wiener measure $Z(\cdot)$ in $\left(\mathbb{R}^{n}, \mathfrak{B}^{n}\right)$ such that

$$
\sqrt{2} d Z(\cdot) \stackrel{d}{=} d \widetilde{W}(\cdot)+d \bar{W}(\cdot)
$$


(if we write formally). Thus

$$
\begin{aligned}
Y(t)+V(t) & =\int_{\mathbb{R}^{n}} \frac{J_{n / 2}\left(|\widetilde{u}| t^{1 / n}\right)}{|\widetilde{u}|^{n-\alpha / 2}} d \widetilde{W}(\widetilde{u})+\int_{\mathbb{R}^{n}} \frac{J_{n / 2}\left(|\widetilde{u}| t^{1 / n}\right)}{|\widetilde{u}|^{n-\alpha / 2}} d \bar{W}(\widetilde{u}) \\
& \stackrel{d}{=} \int_{\mathbb{R}^{n}} \frac{\sqrt{2} J_{n / 2}\left(|\widetilde{u}| t^{1 / n}\right)}{|\widetilde{u}|^{n-\alpha / 2}} d Z(\widetilde{u})=\sqrt{2} X(t) .
\end{aligned}
$$

The theorem is proved.

\section{Concluding Remarks}

We proved a limit theorem for random fields whose spectra have singularities at nonzero frequencies. This theorem generalizes the results for random fields with long range dependence (see [1, 5]) whose spectra have singularities at the origin.

Despite that the limit processes in our case are similar to those in the case of long range dependence, passing to the limit as $a \rightarrow 0$ in Theorem 1 does not prove the result. This can be explained by the difference in the nature of singularities in the spectra for these two cases; see Remarks 2 and 3 .

One can prove other results generalizing and explaining the main theorem. In particular, the limit theorems for the functionals

$$
\int_{\mathbb{R}^{n}} f_{n, r t^{1 / n}, a}(x) H_{m}(\xi(x)) d x, \quad m>1,
$$

can be studied, where $H_{m}(\cdot)$ is the $m$ th Chebyshev-Hermite polynomial. These results will be published elsewhere.

Among interesting open problems are

- proving limit theorems for random fields whose spectra have more than one singularity point;

- considering limit theorems for random fields with OR spectra (see [6]);

- obtaining properties of the fields considered in this paper and comparing them with known results for the case of long range dependence.

\section{BIBLIOGRAPHY}

1. N. N. Leonenko and A. V. Ivanov, Statistical Analysis of Random Fields, Kluwer Academic Publishers, Dordrecht-Boston-London, 1989. MR1009786 (90g:62235)

2. M. I. Yadrenko, Spectral Theory of Random Fields, Optimization Software Inc., New York, 1983 (distributed by Springer-Verlag). MR697386 (84f:60003)

3. N. N. Leonenko and A. Ya. Olenko, Tauberian theorems for correlation functions and limit theorems for spherical averages of random fields, Random Oper. Stoch. Eqs. 1 (1993), no. 1, 57-67. MR 1254176 (95a:60068)

4. A. Ya. Olenko, Tauberian and Abelian theorems for random fields with strong dependence, Ukrain. Mat. Zh. 48 (1996), no. 3, 368-382; English transl. in Ukrainian Math. J. 48 (1996), no. 3, 412-427. MR1408658 (97k:60143)

5. N. N. Leonenko, Limit Theorems for Random Fields with Singular Spectrum, Kluwer Academic Publishers, 1999. MR1687092 (2000k:60102)

6. A. Ya. Olenko, Tauberian theorems for random fields with OR asymptotics. II, Teor. Imovirnost. Matem. Statist. 74 (2006), 81-97; English transl. in Theory Probab. Math. Statist. 74 (2007), 93-111. MR2336781 (2008i:60085)

7. G. N. Watson, A Treatise on the Theory of Bessel Functions, Cambridge University Press, 1944. MR.0010746 (6:64a)

8. C. Houdre, Linear Fourier and stochastic analysis, Probab. Theory and Related Fields 87 (1990), 167-188. MR.1080488(92e:60096) 
9. R. L. Dobrushin, Gaussian and their subordinated self-similar random generalized fields, The Annals of Probability 7 (1979), no. 1, 1-28. MR.515810 (80e:60069)

10. P. Major, Multiple Wiener-Itô Integrals, Lecture Notes in Math., vol. 849, Springer, New York, 1981. MR611334 (82i:60099)

Department of Mathematics and Statistics, La Trobe University, Victoria 3086, Australia

E-mail address: a.olenko@latrobe.edu.au

Department of Probability Theory and Mathematical Statistics, Faculty for Mechanics and Mathematics, National Taras Shevchenko University, Academician Glushkov Avenue 2, KIEV 03127, UKRAINE

E-mail address: bklykavka@yahoo.com

Received 31/AUG/2009

Translated by O. KLESOV 